Harald Reiterer*, Roman Rädle, Simon Butscher und Jens Müller

\title{
Blended Library - neue Zugangswege zu den Inhalten wissenschaftlicher und öffentlicher Bibliotheken
}

DOI 10.1515/bfp-2016-0010

Zusammenfassung: Der Beitrag stellt neue Formen des Zugangs zu den Inhalten von Bibliotheken vor. Diese wurden in Zusammenarbeit mit wissenschaftlichen und öffentlichen Bibliotheken im Rahmen zweier Forschungsinitiativen entwickelt. $\mathrm{Zu}$ Beginn jeder Forschungsinitiative wurden eine Reihe empirischer Studien durchgeführt, die wertvolle Erkenntnisse über die Nutzung von Bibliotheken lieferten. Diese Erkenntnisse wurden in die vier Qualitäten intuitiver, kollaborativer, transparenter und kontextueller Zugang überführt. Diese waren Ausgangspunkt für die Entwicklung mehrerer interaktiver Forschungssysteme. Die Herleitung aller vier Qualitäten der Zugänglichkeit werden erläutert und deren Umsetzung in den Forschungssystemen „Blended Shelf“, „TwisterSearch“, „Tiefenrausch“ und „Expedition“ beschrieben. Abschließend werden die mithilfe von Forschungssystemen gewonnenen Erkenntnisse und Erfahrungen unter Betrachtung der vier Qualitäten der Zugänglichkeit diskutiert.

Schlüsselwörter: Online-Recherche; begreifbare Benutzungsschnittstellen; interaktive Tische; öffentliche Displays; Multi-Display-Umgebungen; Mensch-Computer-Interaktion

\section{Blended Library - New Ways to Access the Collections of Scientific and Public Libraries}

Abstract: The article discusses new ways to access library collections based on two joint initiative projects with scientific and public libraries. In the beginning of each scientific initiative, we conducted a number of empirical studies in order to gain valuable insights about users' patterns when accessing library collections. In detail, we distinguish between intuitive, collaborative, transparent, and contextual access. These four qualities served as a basis for the development of interactive systems. In this

*Kontaktperson: Harald Reiterer, harald.reiterer@uni-konstanz.de Roman Rädle, roman.raedle@uni-konstanz.de Simon Butscher, simon.butscher@uni-konstanz.de Jens Müller, jens.mueller@uni-konstanz.de article, we discuss how we derived these four qualities based on our empirical studies and explain their implementation in the inquiring systems "Blended Shelf", "TwisterSearch", "Tiefenrausch", and "Expedition". Finally, we elaborate on insights and experiences gained through the development of our systems, especially how our work feeds back and further informs the four ways to access library collections.

Keywords: Search and information seeking; tangible user interfaces; table tops; public displays; multi-display environments; human-computer interaction

Inhalt

1 Zugänglichkeit zu Bibliotheken . . . . . . . . 7

2 Neue Interaktionskonzepte für die Bibliothek der

Zukunft ................... 9

2.1 Intuitiv . . . . . . . . . . . . . . . . . 10

2.2 Kollaborativ .................. 11

2.3 Transparent .................. 12

2.4 Kontextuell................... 13

3 Intuitiver Zugang durch Erhalt von Vorzügen der

Recherche in physischen Bibliotheken bei gleichzeitiger Nutzung der Vorteile einer OnlineRecherche ................... 13

4 Unterstützung von kollaborativen Recherchen .. 15

5 Steigerung der Transparenz des Rechercheprozesses ................. 16

6 Kontextualisierung von Bibliotheksdaten mit digitalen Daten des Alltags als neuartiger Einstieg in den Rechercheprozess . . . . . . . . . . . . . 17

7 Resümee .................... 18

\section{Zugänglichkeit zu Bibliotheken}

Bibliotheken und Archive sind die Institutionen unserer Gesellschaft zur Organisation und Aufrechterhaltung unserer kollektiven Wissensspeicher (Reiterer 2015). Die Aneignung von Wissen erfordert den einfachen Zugang zu den jeweiligen Medienbeständen. Trotz fortschreitender 
Digitalisierung werden Medien, auch heute noch, typischerweise in analoger (Regalen, Vitrinen etc.) und digitaler Form (Datenbanken, digitalen Bibliotheken etc.) aufbewahrt. Von deren Koexistenz ist auch noch für eine geraume Zeit auszugehen.

Um den Zugang zu der großen Medienfülle überhaupt möglich zu machen, wurden im Laufe der Jahre umfassende Kataloge als Sammlung von Metadaten entwickelt, die das jeweilige Werk möglichst umfassend charakterisieren und damit wieder auffindbar machen. Diese Kataloge haben sich in den letzten Jahrzehnten von analogen Zettelkästen (Abb. 1) zu funktional immer umfangreicheren Online Public Access Catalogues (OPACs) weiterentwickelt. Letzter Stand sind Ressource Discovery Systeme (RDS) (Abb.2), die nicht nur im lokalen Bestand, sondern weltweit in Online-Katalogen suchen. Zusätzlich bieten sie häufig eine Integration zusätzlicher Datenquellen (z.B. Rezensionen) sowie umfassende Filtermöglichkeiten (z. B. nach Facetten) und eine nach Relevanz sortierte Treffermengendarstellung. Ein Ziel dieser Entwicklung war es, durch entsprechende Gestaltung der Benutzungsoberfläche solcher Online-Kataloge, dem Bibliotheksbesucher einen vertrauten Zugang $\mathrm{zu}$ den analogen und digitalen Medien zu gewähren, indem man sich die von vielen Benutzern gemachte Erfahrung im Umgang mit Web-Suchmaschinen zum Vorbild genommen hat.

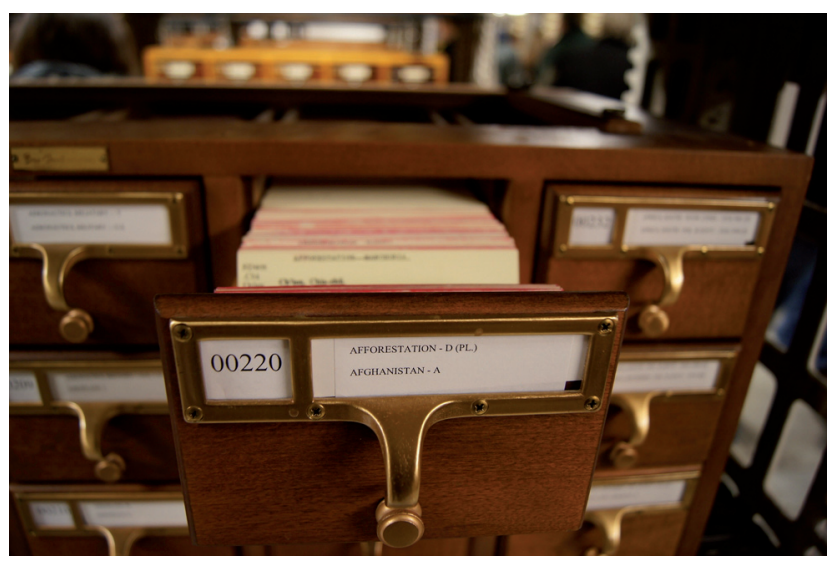

Abb. 1: Analoger Zettelkasten in der Library of Congress in Washington DC, USA

Dies sind Schritte in die richtige Richtung, da eine der großen Herausforderungen für die zukünftige Nutzung unserer Bibliotheken darin besteht, auch weiterhin möglichst allen Mitgliedern unserer Gesellschaft Zugang zu den rapide wachsenden Medienbeständen zu ermöglichen. Dabei spielt die Gestaltung der Benutzungsoberfläche der OnlineKataloge eine zentrale Rolle, denn sie stellt für den Nutzer oft den einzigen Weg zu den Inhalten dar. Vor allem

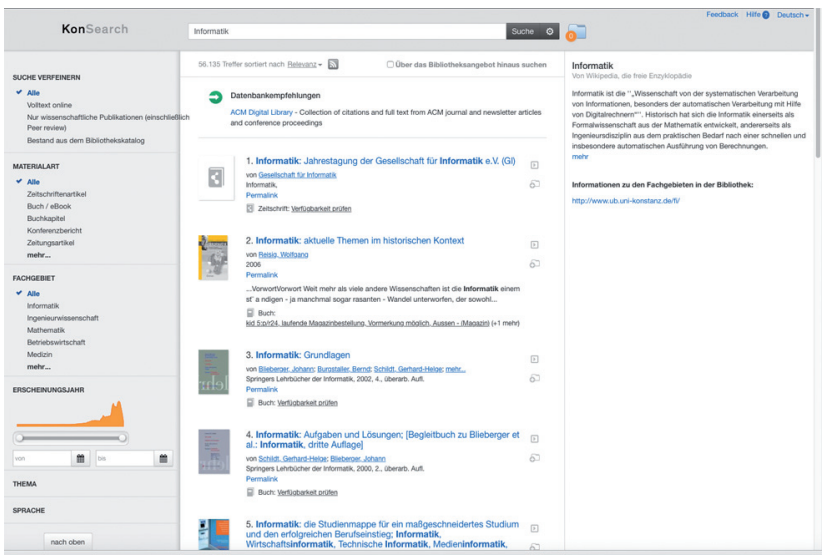

Abb. 2: KonSearch, ${ }^{1}$ die Literatursuchmaschine der Universität Konstanz, auf der Basis von Summon ${ }^{2}$

die Natürlichkeit der Interaktion ist für die einfache $\mathrm{Zu}$ gänglichkeit erfolgsentscheidend.

Die Forschung hat sich zwar in den letzten Jahren diesem Thema verstärkt zugewandt und unter Begriffen wie „Human-Computer Information Retrieval“ (Marchionini, 2006), „Search User Interfaces“ (Hearst, 2009) oder „Blended Library“ (Heilig et al., 2011) eine Reihe von Forschungsanstrengungen $\mathrm{zu}$ diesem Thema beschrieben, doch im Vergleich zu anderen Themengebieten der Informatik ist dieses Forschungsgebiet insgesamt eher schwach entwickelt. Daher wurde der Zugang zu unseren digitalen Inhalten als eine der „Grand Challenges“ unter der Bezeichnung „Erhalt des digitalen Kulturerbes“3 ${ }^{\text {“ }}$ von der Gesellschaft für Informatik (GI) identifiziert und soll deshalb in Zukunft verstärkt in den Fokus der Informatikforschung gestellt werden (Eibl; Löbel; Reiterer 2015).

Bedauerlicherweise haben es bis jetzt nur wenige Systeme aus dem Forschungskontext in die Alltagsnutzung geschafft. Neben einer überschaubaren Menge an aktiven Forschern (vor allem in Deutschland) trägt zu diesem Umstand auch bei, dass sich die Anbieter kommerzieller Recherchesysteme nicht gerade durch Innovationsfreudigkeit hervortun. Dies ist aus unserer Sicht eine grobe Fehleinschätzung. Gerade Bibliotheken bieten eine interessante Anwendungsdomäne, um neue Informatikkonzepte und im Besonderen innovative Interaktionskonzepte mit einer sehr heterogenen Benutzergruppe zu erproben. Aus unserer Sicht ist das komplexe Thema Zugänglichkeit

1 http://www.ub.uni-konstanz.de/konsearch.

2 http://www.proquest.com/products-services/The-Summon-Servic e.html.

3 https://www.gi.de/themen/grand-challenges-der-informatik/digi tale-kultur.html. 
nicht allein mit der Anwendung leistungsfähiger Suchmaschinen erledigt.

Vielmehr geht es um die Entwicklung neuer bzw. verbesserter Qualitäten des Zugangs. Unsere langjährige Forschung im Bereich der Entwicklung von neuen Benutzungsschnittstellen für Bibliotheken (Gerken et al. 2009, Heilig et al. 2011) haben zu der Einsicht geführt, dass die folgenden vier Qualitäten den Zugang reichhaltiger gestalten können:

- Intuitiver Zugang: Durch das Kombinieren der Vorteile der Recherche in physikalischen Bibliotheken und Medien mit den Vorteilen der digitalen Recherche entsteht ein als natürlich empfundener Zugang.

- Kollaborativer Zugang: Durch die Unterstützung von Recherchen in Gruppen werden völlig neue Formen des gemeinsamen Zugangs zu den Inhalten unserer Bibliothek möglich.

- Transparenter Zugang: Durch eine „be-greifbare“ und zugleich visuelle Präsentation des Rechercheprozesses und der Ergebnisse entsteht eine neue Transparenz, die zu einem tieferen Verständnis bzgl. des Rechercheprozesses führt und damit dem Benutzer eine bessere Kontrolle über die erzielten Ergebnisse ermöglicht.

- Kontextueller Zugang: Durch die Verbindung des Bibliotheksbestandes mit Daten unseres (digitalen) Alltags werden neue Zugänge geschaffen und gleichzeitig werden die Bibliotheksinhalte in den Alltagskontext gestellt.

\section{Neue Interaktionskonzepte für die Bibliothek der Zukunft}

Im Rahmen der Forschungsprojekte „Blended Library““ (gefördert vom Ministerium für Wissenschaft, Forschung und Kunst, Baden-Württemberg.) sowie „Lernort Bibliothek“5 (gefördert vom Ministerium für Familie, Kinder, Jugend, Kultur und Sport, Nordrhein-Westfalen) wurden innovative Interaktionskonzepte, unter besonderer Berücksichtigung der oben beschriebenen Qualitäten der

4 Siehe http://hci.uni-konstanz.de/blendedlibrary. Partner: Universität Konstanz, Institut für Wissensmedien in Tübingen und die Bibliotheken der Universitäten Konstanz und Tübingen sowie der ZHAW Winterthur.

5 Siehe http://hci.uni-konstanz.de/libros. Partner: Universität Konstanz und die Stadtbibliothek Köln gemeinsam mit den Pilotbibliotheken des Modelprojektes „Lernort Bibliothek“ des Landes NordrheinWestfalen.
Zugänglichkeit, umgesetzt. Diese neuen Interaktionskonzepte basieren auf dem von uns entwickelten Interaktionsparadigma „Blended Interaction“ (Reiterer, 2014). Es verfolgt das Ziel, die Interaktion mit dem Computer an unseren Erfahrungen der Interaktion mit der realen, nicht digitalen Welt zu orientieren und so natürlicher zu machen.

Anhand von ausgewählten Beispielen aus diesen beiden Vorhaben soll hier exemplarisch gezeigt werden, welche neuen Interaktionskonzepte zur Steigerung der $\mathrm{Zu}$ gänglichkeit für die Bibliothek der Zukunft relevant sein könnten. Die oben beschriebenen vier neuen Qualitäten des Zugangs waren der Ausgangspunkt zur Entwicklung neuer interaktiver Zugangsformen. Diese Qualitäten basieren auf eigenen langjährigen Erfahrungen im Zusammenhang mit der Entwicklung von Recherchesystemen und umfassenden empirischen Erhebungen, die wir im Rahmen der beiden Vorhaben an der Bibliothek der Universität Konstanz und in mehreren Bibliotheken der Stadt Köln durchgeführt haben.

Dem Prinzip des „User-Centered Design“ folgend, wurden die Bibliotheknutzer sowie die Bibliothekare, als die beiden wichtigsten Zielgruppen, schon frühzeitig in den Entwicklungsprozess mit einbezogen. Das User-Centered Design ist die gängige Methode beim User Experience Design (Hartson und Pyla, 2012) und wird fortwährend im Design-, Entwicklungs- und Evaluationsprozess angewandt, um einerseits Aufgaben, Ziele und Probleme potentieller Benutzer zu identifizieren und daraus Bedürfnisse und Anforderungen abzuleiten, und um andererseits die Erfüllung der Anforderungen zu überprüfen.

Um diese Bedürfnisse und Probleme der Bibliotheksnutzer identifizieren und daraus Anforderungen ableiten zu können, wurden im Umfeld wissenschaftlicher Bibliotheken Online-Befragungen zu Recherche- und Leseverhalten durchgeführt. Ein Fragebogen zu Gruppenarbeitssituationen wurde an Studierende der Universität Konstanz ausgeteilt und ausgewertet sowie darauf basierende Einzelinterviews mit weiteren Studierenden zu Gruppenarbeitssituationen geführt. Zur Anforderungserhebung in öffentlichen Bibliotheken wurde eine mehrtägige, teilnehmende Beobachtung in drei Bibliotheksstandorten der Stadtbibliothek Köln durchgeführt.

Im Folgenden werden einige ausgewählte Ergebnisse vorgestellt, die zur Herleitung der neuen Qualitäten intuitiver, transparenter, kollaborativer und kontextueller $\mathrm{Zu}$ gang führten. Tab. 1 zeigt die Zuordnung der vier Qualitäten zu den Forschungssystemen, die im Rahmen beider Projekte „Blended Library“ und „Lernort Bibliothek“ entstanden sind. Aus Platzgründen wird in diesem Artikel jeweils nur ein Forschungssystem pro Qualität vorgestellt 


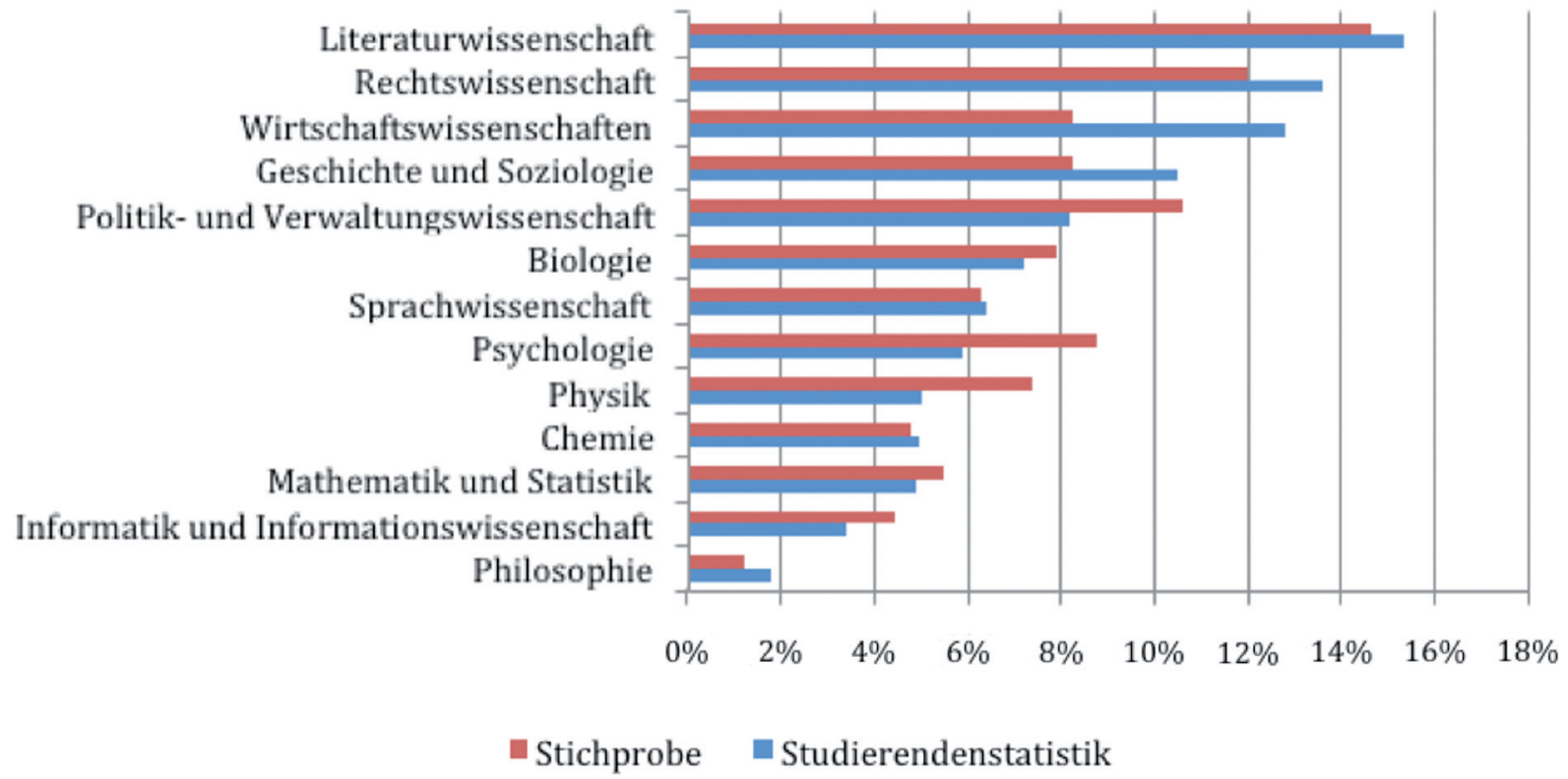

Abb. 3: Vergleich der gemessenen Stichprobe zur Gesamtpopulation unterteilt in die 13 Fachbereiche der Universität Konstanz

(in Tab. 1 unterstrichen). Die in der Tabelle angegebenen Weblinks verweisen auf weiterführende Literatur oder Videos der einzelnen Systeme.

Tab. 1: Zuordnung der neuen Qualitäten zu Forschungssystemen, die im Rahmen der beiden Projekte „Blended Library“ und „Lernort Bibliothek" entstanden sind

\begin{tabular}{|c|c|}
\hline Qualität & Forschungssystem \\
\hline Intuitiver Zugang & $\begin{array}{l}\text { Blended Shelf } \\
\text { http://www.blendedlibrary.org/blendedshelf/ } \\
\text { Hybrid Bookshelf (kommerziell) } \\
\text { http://www.hybridbookshelf.de } \\
\text { Integrative Workplace } \\
\text { http://www.blendedlibrary.org/integrative } \\
\text { workplace/ }\end{array}$ \\
\hline $\begin{array}{l}\text { Kollaborativer } \\
\text { Zugang }\end{array}$ & $\begin{array}{l}\text { TwisterSearch } \\
\text { http://www.blendedlibrary.org/twistersearch/ } \\
\text { Facet Search } \\
\text { http://www.blendedlibrary.org/facetsearch/ }\end{array}$ \\
\hline $\begin{array}{l}\text { Transparenter } \\
\text { Zugang }\end{array}$ & $\frac{\text { Tiefenrausch }}{\text { http://hci.uni-konstanz.de/libros }}$ \\
\hline $\begin{array}{l}\text { Kontextueller } \\
\text { Zugang }\end{array}$ & $\frac{\text { Expedition }}{\text { http://hci.uni-konstanz.de/libros }}$ \\
\hline
\end{tabular}

\subsection{Intuitiv}

Durch das Kombinieren aller Vorteile der Recherche in physischen Bibliotheken und Medien mit den Vorzügen der digitalen Recherche entsteht ein als natürlich empfundener Zugang. Diese Erkenntnis basiert einerseits auf unserer Forschung zum Bereich „Blended Interaction“ (Reiterer 2014) und wurde andererseits durch die Ergebnisse einer Benutzerbefragung in der Bibliothek der Universität Konstanz gewonnen. Dazu wurde ein OnlineFragebogen per E-Mail an alle studentischen und akademischen Mitglieder der Universität Konstanz gesandt und in gedruckter Form in der Bibliothek ausgelegt (Erhebungszeitraum 2011). Der Fragebogen war über drei Monate online bzw. in der Bibliothek zugänglich und bestand neben Fragen zur Demographie vor allem aus Fragen zum Rechercheverhalten sowie zu Lese- und Schreibprozessen.

An der Befragung haben insgesamt 682 Mitglieder der Universität Konstanz teilgenommen. Davon waren 599 Studierende und 83 akademische Mitarbeiter. Verglichen mit der Studierendenstatistik der Universität Konstanz aus demselben Zeitraum konnte so eine Rücklaufquote von $5,63 \%$ erzielt werden. Die Stichprobe ist verglichen mit der Gesamtpopulation repräsentativ in Bezug auf Zuordnung zu Fachbereich, Geschlecht und angestrebtem Abschluss (Abb. 3).

Die Bibliothek der Universität Konstanz ist eine Freihandbibliothek. Diese erlaubte den Nutzern auch ein „Suche am Regal“. Der dabei entstehende „SerendipityEffekt“ (Björneborn, 2008) führt oft zu interessanten Zufallsfunden und wird von den Benutzern sehr geschätzt (siehe Abb.3). Außerdem hilft die Orientierung im realen 
Raum, sich einen schnellen und besseren Überblick über große Inhalte zu verschaffen.

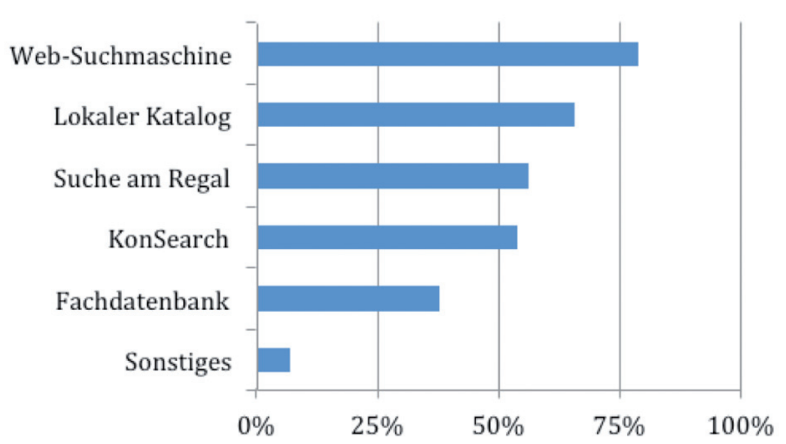

Abb. 4: Auswertung der Frage „In welchen Katalogen/Suchmasken suchen Sie nach weiterführender Literatur?“ (mehrfach Nennung möglich) ${ }^{6}$

Die Herausforderung, einen intuitiven Zugang zu den digitalen Inhalten zu schaffen, besteht nun darin, die Vorteile der „Suche am Regal“ mit jenen der digitalen Recherche zu kombinieren. In Kapitel 3 zeigen wir anhand des Forschungsprototyps „Blended Shelf“, wie dies umgesetzt werden kann. Als digitales Bücherregal orientiert es sich an real-weltlichen Eigenschaften von Regalen und Büchern (beispielsweise deren Form, Größe oder Farbe). Gleichzeitig erweitert es aber die realweltliche Recherche mit digitalen Funktionen wie Suchen, Filtern und Sortieren.

\subsection{Kollaborativ}

Heute werden in Bibliotheken in der Regel nur Systeme angeboten, die die Recherche des Einzelnutzers unterstützen. Dazu gibt es zahlreiche Untersuchungen, die die Bedeutung der kooperativen Recherche für die Praxis belegen (Morris, 2013). Erstaunlicherweise gibt es dazu aber noch wenige Forschungsarbeiten. Insbesondere durch die Unterstützung von Recherchen in Teams werden völlig neue Formen des gemeinsamen Zugangs zu den Inhalten der Bibliothek möglich. Die oben genannte Befragung hat einen großen Bedarf an Gruppenarbeitsplätzen und Räumen gezeigt, da der studentische Alltag heute vielfach durch Teamarbeit geprägt ist.

Um weitergehende Erkenntnisse zu Gruppenarbeit zu gewinnen, wurden im Rahmen einer nachfolgenden Erhebung ein Fragebogen ( $n=18$ ) zur Gruppenarbeit ausgege-

6 KonSearch (RDS) wurde erst im Mai 2011 eingeführt. ben und Einzelinterviews ( $\mathrm{n}=20)$ mit Studierenden zum Thema Gruppenarbeit durchgeführt. In beiden Fällen wurden nur Studierende höherer Semester befragt, weil diese in der Regel bereits ausreichend Erfahrung mit Gruppenarbeit gesammelt haben. Wir waren insbesondere an deren Erfahrungen mit gruppendynamischen Prozessen während der wissenschaftlichen Arbeit und Recherche interessiert. Beispielsweise wurden die Teilnehmer befragt wann und wozu sie Gruppenarbeit anwenden (Abb.5) und welche Probleme dabei auftreten, welche Materialen und Hilfsmittel sie verwenden und was ihre angestrebten Ziele bei der Gruppenarbeit sind (Abb. 6).

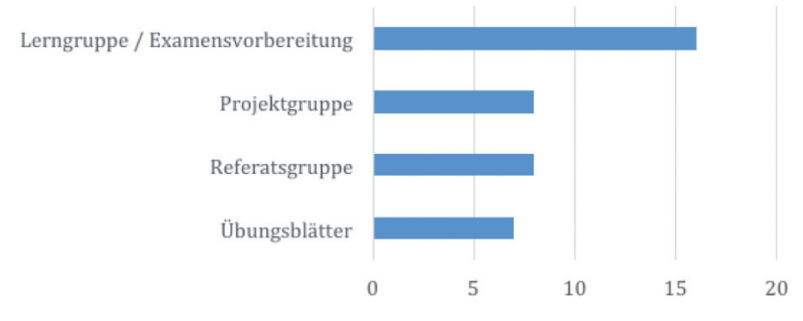

Abb. 5: Gruppenarbeitssituationen mit Kommilitonen während des Studiums (Mehrfachnennungen möglich) ${ }^{7}$

Der häufigste Grund für Gruppenarbeit ist gemeinsames Lernen und Vorbereitung auf Klausuren oder Examina gefolgt von Projekt- und Referatsarbeiten. Als Vorteil der Gruppenarbeit gegenüber Einzelarbeit wurden der Austausch und die Diskussion mit anderen Gruppenmitgliedern genannt. Wichtig war auch der Aspekt gegenseitigen Erklärens. Projektgruppen und Referatsgruppen wurden häufig zur Planung, Strukturierung und Aufgabenverteilung gebildet und gegenüber Einzelarbeit wegen der besseren Effizienz und Effektivität bevorzugt.

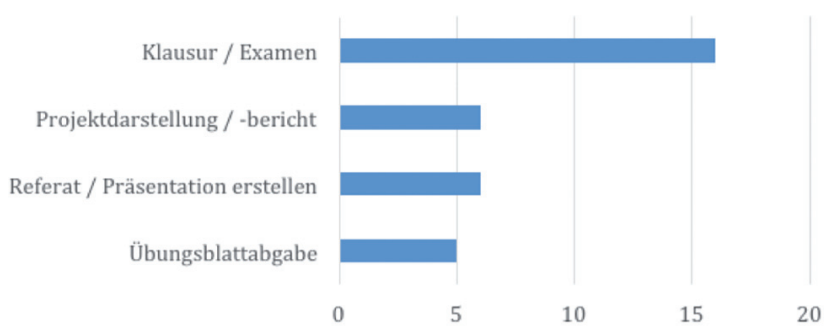

Abb. 6: Das angestrebte Ziel der Gruppe (Mehrfachnennungen mög(ich) ${ }^{8}$

7 Es sind nur Kategorien aufgeführt mit mehr als zwei Nennungen. 8 Es sind nur Kategorien aufgeführt mit mehr als zwei Nennungen. 
Aus den Erkenntnissen der Befragung leiteten wir als weitere Herausforderung die Schaffung eines kollaborativen Zugangs zu den Bibliotheksinhalten ab. Rechercheziele sollten gemeinsam definiert und strukturiert werden können. Rechercheaufgaben sollten verteilt und gesammelte Information aus verschiedenen Quellen (z. B. aus dem Internet, lokalen Katalogen, Büchern oder Zeitschriften) in einem gemeinsamen Arbeitsbereich gebündelt werden, um für alle gleichermaßen parallel zugänglich zu sein. Im Kapitel 4 zeigen wir anhand des Forschungssystems „TwisterSearch“ wie eine kollaborative Internet-Recherche realisiert werden könnte.

\subsection{Transparent}

Durch eine im doppelten Wortsinn „be-greifbare“ sowie visuelle Präsentation des Rechercheprozesses und der Ergebnisse entsteht eine neue Transparenz, die zu einem tieferen Verständnis bzgl. des Rechercheprozesses führt und damit dem Benutzer eine bessere Kontrolle über die erzielten Ergebnisse ermöglicht.

Ein für den Bibliotheksnutzer transparenter Suchprozess macht es einfacher, die Suche anzupassen bzw. auf Basis der Treffermenge neu zu definieren. Die Bedeutung einer transparenten Recherche wurde unter anderem bei der Erhebung von Anforderungen im Umfeld öffentlicher Bibliotheken deutlich. Mithilfe von Beobachtungsinterviews wurden detaillierte Informationen über die Arbeitsweise von Besuchern und Bibliothekaren erhoben, z.B. welche Schritte sie bei ihrem Besuch bzw. der Recherche durchlaufen und welche Hilfsmittel und Systeme sie dabei nutzen. Der Fokus der Erhebung lag auf dem Kontext der innerhalb der Bibliothek genutzten Systeme (OPAC, physische Regale, persönliche Beratung). Innerhalb der einwöchigen Erhebung wurden insgesamt 99 Bibliotheksbesucher und Bibliothekare bei ihren Recherchepraktiken innerhalb von drei öffentlichen Bibliotheken beobachtet und befragt (Tab. 2).

Tab. 2: Anzahl der Befragungen in der Stadtbibliothek (zentral, zwei Niederlassungen)

\begin{tabular}{|c|c|c|c|c|}
\hline & Bibliothekare & Benutzer & $\begin{array}{l}\text { Schüler (Metho- } \\
\text { dentraining) }\end{array}$ & Gesamt \\
\hline $\begin{array}{l}\text { Roden- } \\
\text { kirchen }\end{array}$ & 2 & 7 & & 9 \\
\hline Mülheim & 2 & 10 & & 12 \\
\hline Zentrale & 3 & 19 & 56 & 78 \\
\hline Gesamt & 7 & 36 & 56 & 99 \\
\hline
\end{tabular}

Als wesentliches Motiv für den Besuch in einer öffentlichen Bibliothek wurde die Recherche nach bestimmten Büchern oder Themen genannt, d.h., der typische Besucher kommt schon mit einem konkreten Suchbedürfnis. Teilweise wurde von den Benutzern bereits zuhause eine Vorrecherche durchgeführt (im Web) und die Ergebnisse wurden mithilfe von Zetteln oder dem Smartphone mit in die Bibliothek gebracht. Im Falle der Katalogrecherche wurde beobachtet, dass Besucher, die im Katalog nicht fündig wurden, oft zurück ans Regal gingen und dort in das Lesen der Buchrücken verfielen. Das größte Problem der Benutzer beim Recherchieren lag im Formulieren einer geeigneten Suchanfrage. Dies wurde dadurch verschärft, dass die Recherche in den Online-Katalogen der Bibliotheken anderen Regeln unterliegt als die Recherche im Internet. Während im Internet die Suchanfrage sehr spezifisch und detailliert gestellt werden muss, um auf eine überschaubare Trefferzahl zu gelangen, ist es in der Bibliothek aufgrund des kleineren Bestandes nötig, nach übergeordneten Begriffen und Themengebieten zu suchen. Dies ist vor allem einer häufig fehlenden Volltextindizierung geschuldet. Dies führt aber dazu, dass Suchanfragen häufig zu leeren Ergebnismengen führen, ohne dass die Nutzer den Grund dafür nachvollziehen können. Dies hat zur Folge, dass die Bibliotheksbenutzer sich an das Bibliothekspersonal wenden und um Unterstützung bei der Recherche bitten müssen. Diese Unterstützung erfolgt dann in der Regel dergestalt, dass der Bibliothekar eigenständig unter Nutzung des OPACs die Recherche durchführen und dem Benutzer die Resultate mitteilen wird. Dabei besteht aber für den Benutzer keine Möglichkeit, etwas hinsichtlich geeigneter Recherchestrategien oder Nutzung des OPACs zu lernen. Dies wird dadurch erschwert, dass heute übliche Desktop-PCs mit einem Bildschirm sowie mit Tastatur und Maus für Beratungsszenarien denkbar schlecht geeignet sind. Vielfach konnte auch beobachtetet werden, dass Benutzer gleich direkt zur Beratung gegangen sind und auf Nachfrage erklärten, dass ihre schlechten Erfahrungen mit dem lokalen Online-Katalog sie gleich die „Abkürzung“ zur Beratung nehmen ließ.

Diese Erhebung verdeutlicht, dass die Transparenz des Suchprozesses ein entscheidendes Kriterium für eine erfolgreiche Recherche in Bibliotheken darstellt. Nur wenn der Suchprozess und das Zustandekommen der Ergebnismenge bzw. der Grund für eine leere Ergebnismenge verstanden wird, kann eine adäquate Anpassung der Suchkriterien an die Gegebenheiten des Kataloges vorgenommen werden. Im Kapitel 5 zeigen wir anhand des Forschungssystems „Tiefenrausch“ wie ein transparenter Rechercheprozess realisiert werden könnte. 


\subsection{Kontextuell}

Durch die Verbindung des Bibliotheksbestandes mit Daten unseres (digitalen) Alltags werden neue Zugänge geschaffen und zugleich die Bibliotheksinhalte in den Alltagskontext gestellt.

Der Medienbestand in heutigen öffentlichen Bibliotheken umfasst weitaus mehr als nur physische Bücher. Bibliotheken bieten ein breites Medienangebot, welches von E-Books, über Zeitschriften bis hin zu Musik, in Form von CDs, und Filmen, in Form von DVDs, reicht. Jedoch bietet dieser Medienbestand nur selten Verknüpfungen zu der Vielzahl an Datenquellen unserer digitalen Welt.

Die Ergebnisse der einwöchigen Erhebung an drei öffentlichen Bibliotheken in Köln zeigten, dass externe Informationsquellen häufig als Inspiration für Recherchen herangezogen werden, z. B. Buchempfehlungen von Freunden oder Bekannten, aber auch aktuelle Themen aus Zeitungen, Rundfunk, Fernsehen, Sozialen Medien oder dem Web.

Bei der Einschätzung der Relevanz eines gefunden Mediums spielen externe Informationen ebenfalls eine wichtige Rolle. Heute sind die Leser vielfach auf die Informationen auf dem Buchrücken angewiesen. Weiterführende Informationen müssen sie selbst - beispielsweise durch Nutzung eines Smartphones, Tablets oder eines in der Bibliothek verfügbaren PCs - recherchieren. Heute steht eine Reihe von externen digitalen Quellen zur Verfügung, die man in den digitalen Rechercheprozess integrieren könnte. Neben Rezensionen aus digitalen Quellen wie „Library Thing for Libraries"9 oder Sozialen Medien (z.B. Facebook, Twitter) sind hier auch weiterführende Informationen zum Autor (z.B. aus Wikipedia) hilfreich für die Auswahlentscheidung.

Die Kontextualisierung von digitalen Datenquellen bietet daher unterschiedliche Potenziale: Zum einen kann eine Verknüpfung des Bibliotheksbestandes mit digitalen Quellen außerhalb der Bibliothek einen neuen Einstieg in den Medienbestand bieten, zum anderen können die vorhandenen Medien mit zusätzlichen Informationen angereichert werden, um eine bessere Einschätzung der Relevanz für die persönlichen Interessen zu ermöglichen. Im Kapitel 6 zeigen wir anhand des Forschungssystems „Expedition" wie eine Integration externer Datenquellen in den Rechercheprozess realisiert werden könnte.

Unbestritten sind die hier vorgestellten neuen Qualitäten der Zugänglichkeit erst ein Anfang und wir können hier sicher nicht den Anspruch einlösen, eine vollständige

9 https://www.librarything.com.
Sammlung von erfolgsversprechenden Qualitäten der $\mathrm{Zu}$ gänglichkeiten für die Bibliothek der Zukunft vorzulegen. In den folgenden vier Kapiteln werden eine Reihe von Forschungssysteme wie „Blended Shelf“, „TwisterSearch“, „Tiefenrausch“ und „Expedition“ vorgestellt und der Bezug zu den vier Qualitäten intuitiver, kollaborativer, transparenter und kontextueller Zugang hergestellt. Diese Systeme verstehen sich als ein erstes „Proof of Concept“ und sollen die mit den Qualitäten verbundenen Möglichkeiten anschaulich machen.

\section{Intuitiver Zugang durch Erhalt von Vorzügen der Recherche in physischen Bibliotheken bei gleichzeitiger Nutzung der Vorteile einer Online-Recherche}

Wie die Erhebungen in Kapitel 2.1 gezeigt haben, wird das realweltliche Stöbern an Bücherregalen von Bibliotheksbenutzern aufgrund seines intuitiven und unmittelbaren Zugangs sehr geschätzt und bietet darüber hinaus auch noch die Möglichkeit für interessante Zufallsfunde.

Das Forschungssystem „Blended Shelf“ (Kleiner et al. 2013) vermischt die Vorzüge der Recherche in physischen Bibliotheken mit jenen der digitalen Recherche (siehe Abb. 8). Der Benutzer kann ein 3D-Bücherregal durch Touch-Interaktion steuern. Dabei wird das herkömmliche Regalbrowsen durch eine naturalistische Darstellung der Medien (z. B. Originalcover, Dicke etc.) simuliert. Gleichzeitig lassen sich aber auch die Vorzüge der digitalen Recherche nutzen, indem man beispielsweise Filter definieren und kombinieren kann (nach Fachgebieten, Erscheinungsjahr, Farbe des Einbandes etc.) bzw. auch noch zusätzliche oder alternative Suchbegriffe in einem Suchfeld eingeben kann. Durch Filter und Suchbegriffe werden die Inhalte des Regals beschränkt. Bücher können durch Berühren aus dem Regal „genommen“ werden. Dann werden weiterführende Informationen am visuell aufgeklappten Buch angezeigt, wie Katalogdaten, aber auch weiterführende Inhalte aus anderen Quellen wie Rezensionen. Gerade für E-Books erscheint ein derartiger Ansatz vielversprechend, da man ihnen quasi ein „begreifbares“ Erscheinungsbild verleiht und sie damit dem realweltlichen Stöbern zugänglich machen kann. Die vom Benutzer gewählten Bücher können als Favoriten abgespeichert und direkt als digitale Version heruntergeladen werden. Falls das Medium nur analog verfügbar ist, wird der Nutzer mittels QR-Code (lesbar via mobiler Endgeräte) auf eine 

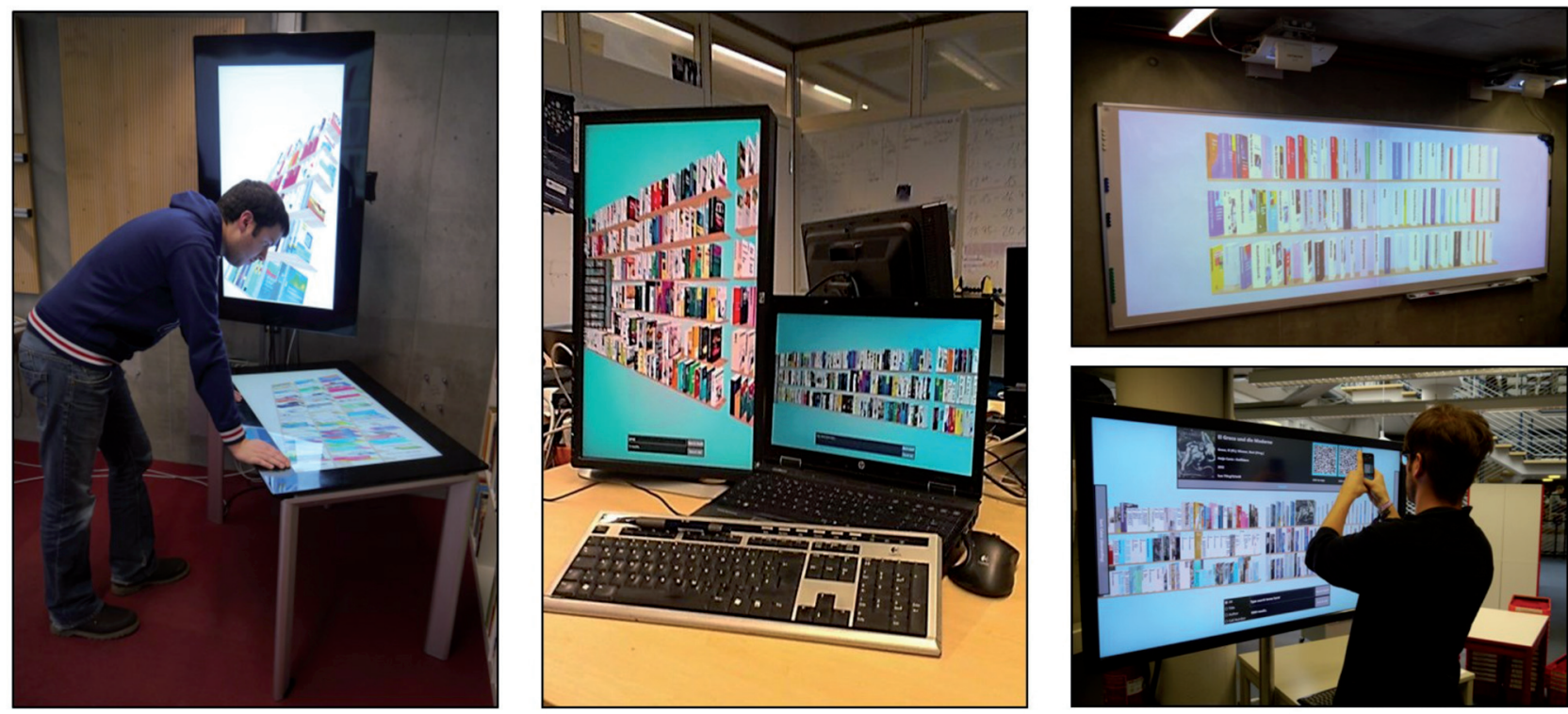

Abb. 8: „Blended Shelf“ im Living Lab und in den Räumen der Bibliothek der Universität Konstanz
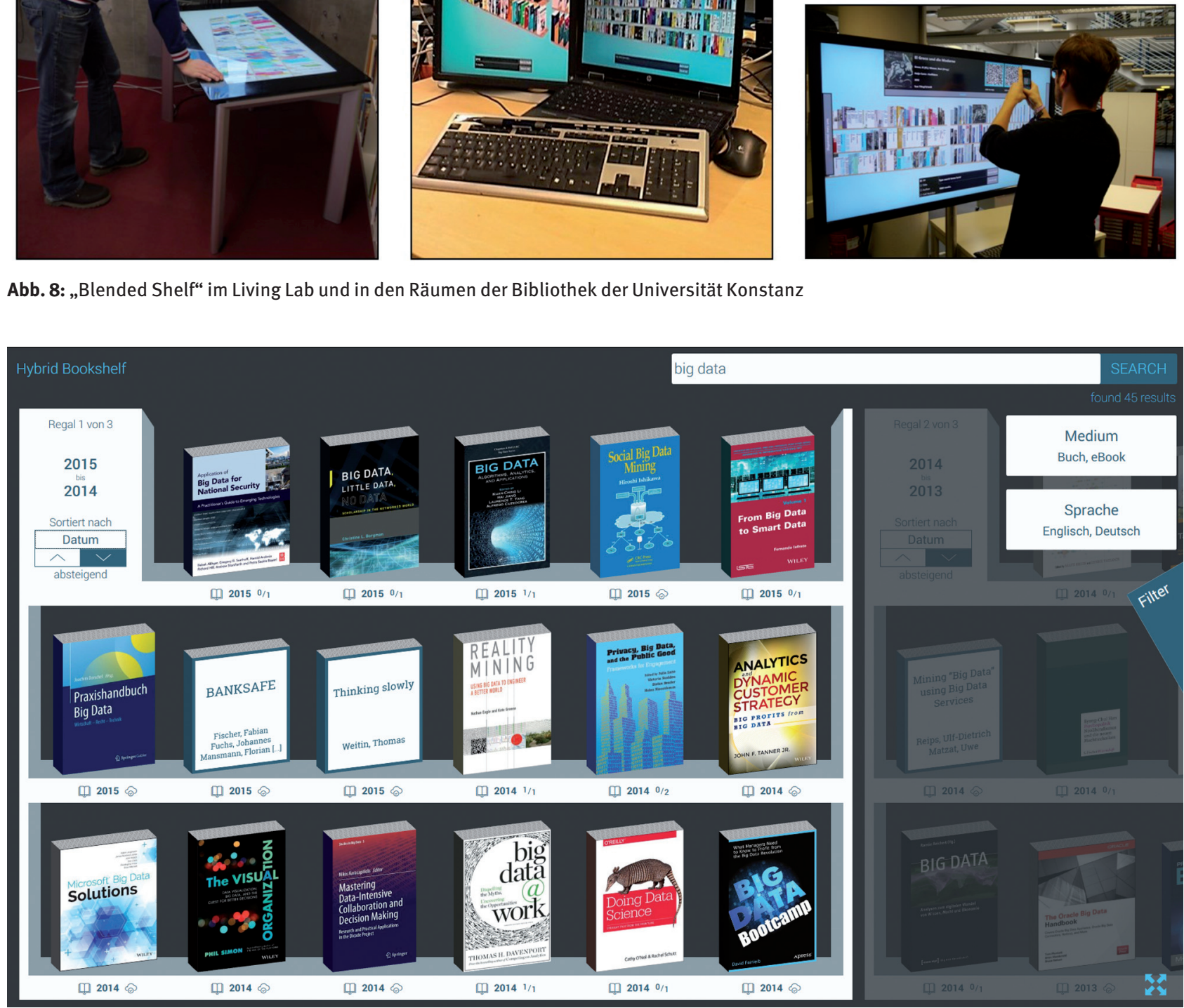

Abb. 9: Ergebnisdarstellung des Hybrid Bookshelf der Bibliothek der Universität Konstanz ${ }^{10}$

Website geleitet (zeigt alle wichtigen Daten wie Signatur, Titel etc., auch Lageplan) bzw. kann davon einen Ausdruck erstellen und wird so zum Freihandregal geleitet.

Die Idee des „Blended Shelf“ wurde von den an der Forschungsinitiative „Blended Library“ beteiligten Biblio-

10 http://www.hybridbookshelf.de. theken sehr gut aufgenommen und daher, unter Federführung der Bibliothek der Universität Konstanz, ${ }^{11} \mathrm{zu}$ einer kommerziellen Version namens „Hybrid Bookshelf“ weiterentwickelt (Kohl-Frey 2014). Diese soll nach einer Erpro-

11 http://www.ub.uni-konstanz.de/bibliothek/projekte/blended-sh elf/. 
bungsphase in Konstanz in einer Reihe weiterer wissenschaftlicher Bibliotheken des Landes Baden Württemberg zum Einsatz kommen. Abb. 9 zeigt die aktuelle Version des „Hybrid Bookshelf“.

\section{Unterstützung von kollaborativen Recherchen}

Wie die Erhebungen in Kapitel 2.2 gezeigt haben, ist Gruppenarbeit eine gängige Praxis in wissenschaftlichen Bibliotheken zur Vorbereitung auf eine Projektpräsentation oder ein Referat. „TwisterSearch“ ist ein Forschungssystem das mit dem Fokus auf eine kooperative Recherche im Internet konzipiert wurde.

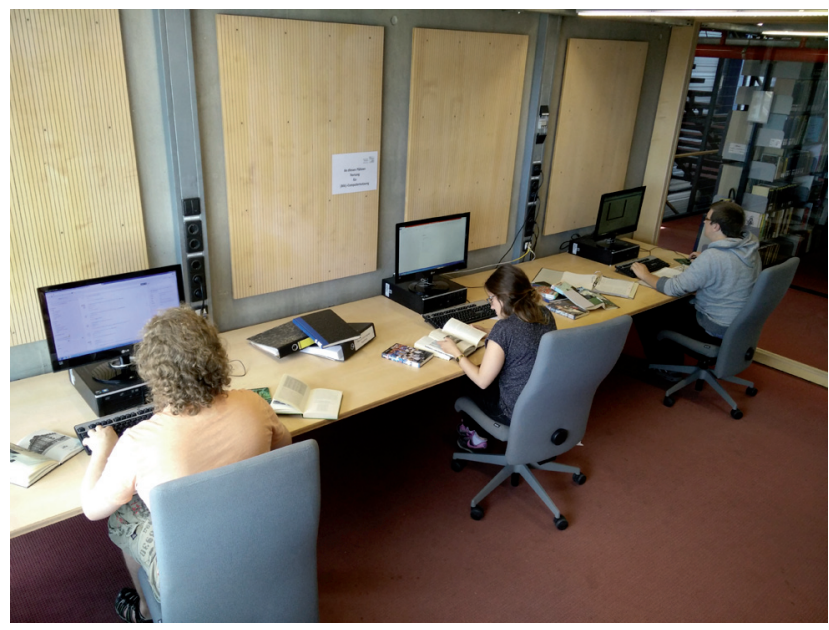

Abb. 10: Gruppenarbeit in der Bibliothek der Universität Konstanz in einem der Gruppenräume

Sobald Inhalte gemeinsam recherchiert werden müssen, sitzen die Studierenden häufig räumlich voneinander getrennt und arbeiten an unterschiedlichen Computern, wie in Abb.10 zu sehen ist. Hierbei ergeben sich zumindest zwei Nachteile, die auch in der Erhebung erkannt wurden. Erstens gehen die natürlichen Qualitäten der Gruppenarbeit verloren, indem, durch die räumliche Distanz, Diskussionen und der damit verbundene Informationsaustausch mit anderen Gruppenmitgliedern erschwert werden. Zweitens arbeiten die Gruppenmitglieder weitgehend unabhängig voneinander und die gefundenen Informationen müssen später mühsam zusammengeführt werden.

Das Ziel von „TwisterSearch“ war es, eine Gruppe bei der Internet-Recherche besser zu unterstützen, indem es die vorher genannten Probleme überwindet. Die Gruppenmitglieder sitzen um einen multitouchfähigen Tabletop

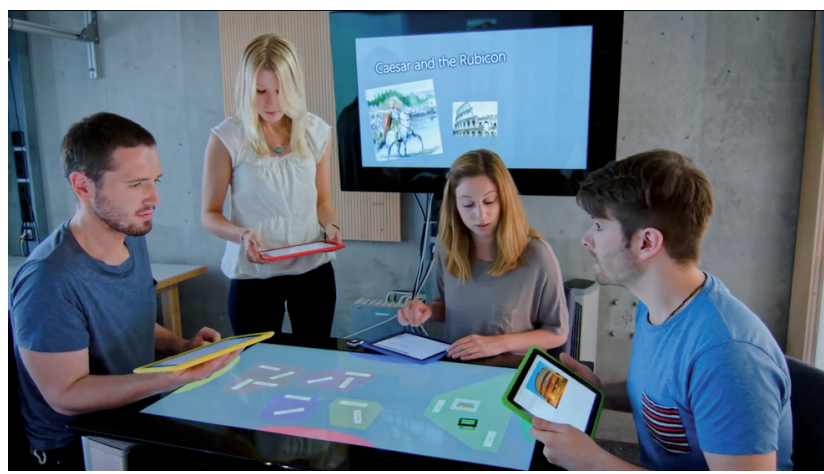

Abb. 11: Eine Gruppe recherchiert im Internet mithilfe von „TwisterSearch“ im Living Lab der Bibliothek der Universität Konstanz

und nutzen zusätzlich Tablets (Abb.11). Die Tablets und der Tisch sind mit dem W-LAN verbunden und kommunizieren miteinander. Die Benutzer können sich jederzeit beraten und wichtige Information austauschen oder einander bei aufgabenspezifischen Problemen helfen. Der in „TwisterSearch“ umgesetzte Arbeitsablauf entspricht dem „Model of Understanding Social Search“ (Evans und Chi 2008). Die Gruppenmitglieder sammeln Stichworte, die für die spätere Suche von Bedeutung sind, geben diese mithilfe der Tablets ein und gruppieren diese anschließend gemeinsam auf dem Tabletop. Die Gruppierung wird durch farbliche Cluster visuell hervorgehoben. Als Resultat dieses Prozesses erhält die Gruppe eine strukturierte Sammlung von Stichworten. Diese Sammlung repräsentiert das gemeinsame Verständnis, zu welchen Sachverhalten weitere Inhalte gefunden werden sollen. Die Suche selbst kann dann anhand der erstellten Cluster aufgeteilt werden. Jedes Gruppenmitglied platziert dazu einen farbigen physischen Baustein (als Repräsentanten der jeweiligen Tablets) in einem Cluster. Die Stichworte werden automatisch auf das jeweilige farblich übereinstimmende Tablet übertragen und an die Suchmaschine Google gesandt. Jedes Mitglied recherchiert somit individuell auf seinem Tablet nach Information für das ihm zugewiesene Cluster und bekommt die Suchergebnisse auf dem Tablet angezeigt. Wird ein Suchergebnis als relevant betrachtet, kann es mit einem Knopfdruck an den Tabletop gesendet und mit den anderen Mitgliedern geteilt werden. Dies geschieht wiederum durch Platzierung von farbigen physischen Bausteinen. Die Suchergebnisse in diesem Cluster werden dann auf allen Tablets synchron angezeigt und können von allen Gruppenmitgliedern bequem gelesen werden. So tragen alle Gruppenmitglieder ihren Teil zum Suchergebnis und der Lösung der Aufgabe bei. Während der Suche können weitere Stichworte hinzugefügt, Suchstrategien besprochen und Ergebnisse präsentiert und diskutiert werden. 

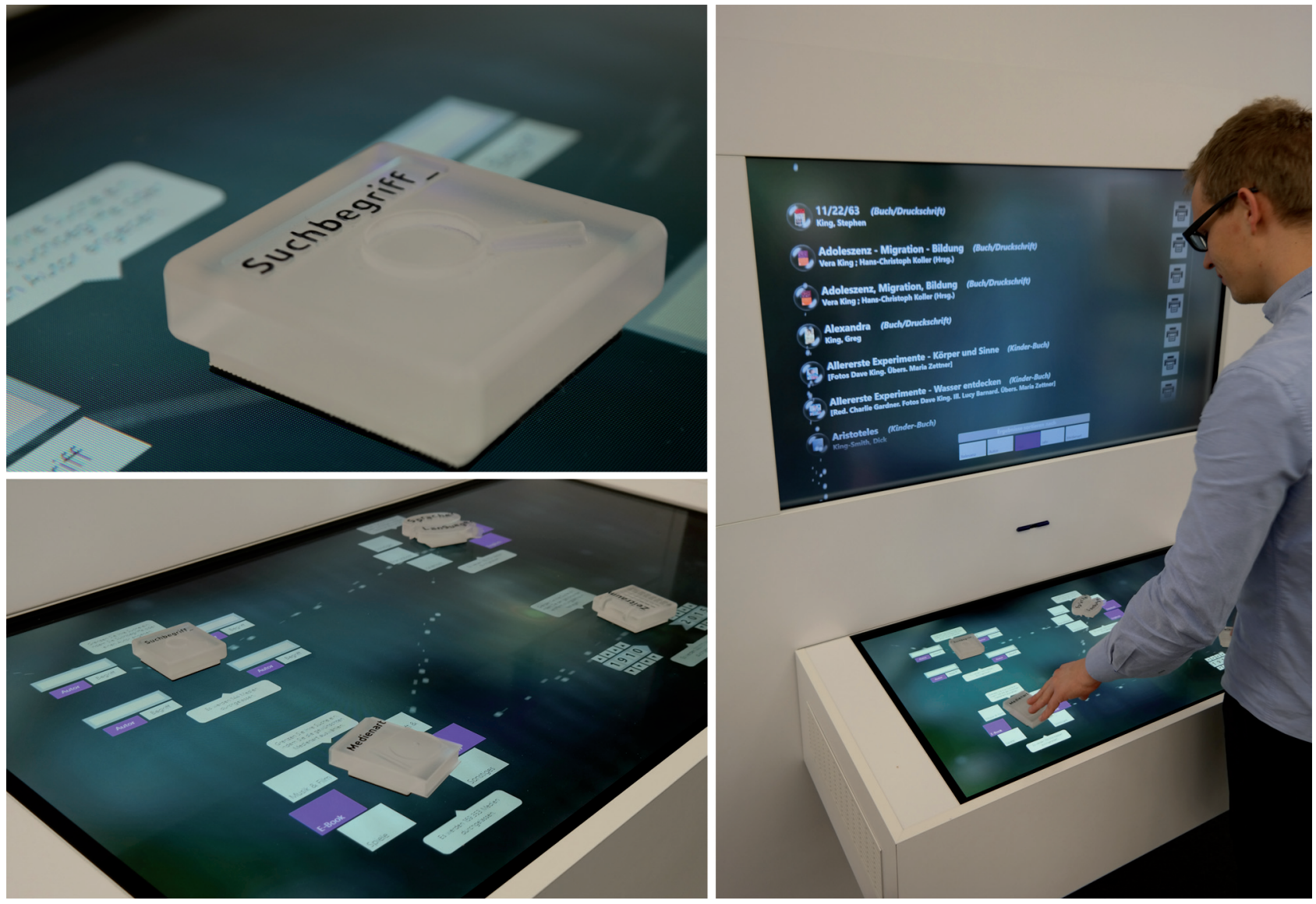

Abb. 12: Transparente Recherche im lokalen Bibliotheksbestand der Stadtbibliothek Köln

„TwisterSearch“ wurde als Werkzeug für die kollaborative Recherche im Internet auch praktisch erprobt. In Rädle; Jetter; Reiterer (2013) werden die Ergebnisse der Erprobung im Detail vorgestellt. Es zeigte sich dabei die prinzipielle Eignung des gewählten technischen Settings für die kollaborative Recherche.

\section{Steigerung der Transparenz des Rechercheprozesses}

Ein transparenter Rechercheprozess erlaubt es, den Nutzern die Suchkriterien an die Ergebnismenge anzupassen und somit die Suche zu verfeinern. Wie die Erhebungen in öffentlichen Bibliotheken zeigen (siehe Kapitel 2.3), besteht gerade bei der Formulierung von Suchanfragen in Online-Katalogen noch erheblicher Unterstützungsbedarf.

Das System „Tiefenrausch“ wurde für öffentliche Bibliotheken entwickelt. Die Installation basiert auf dem Forschungsprototyp „Facet-Streams“ (Jetter et al. 2011). Das Forschungssystem „Tiefenrausch“ realisiert den transparenten Rechercheprozess durch physische Bausteine und das Zusammensetzen dieser Bausteine zu einer visuellen Filterkette. Das System wurde über Monate im Bibliotheksalltag einer öffentlichen Bibliothek erprobt. Die technische Grundlage bildet ein multitouchfähiger Tabletop, welcher, neben einer Toucheingabe, auch die Umsetzung von Tangible User Interfaces (TUIs) erlaubt. Physische Bausteine, die auf dem Tisch platziert sind, werden erkannt und können somit für die Interaktion mit dem System genutzt werden. Beim Tiefenrausch repräsentieren unterschiedliche Bausteine unterschiedliche Filtermöglichkeiten (Medientyp, Sprache oder Veröffentlichungsjahr). Durch das Auflegen eines Bausteins auf den Tisch kann der entsprechende Filter definiert werden und gliedert sich so automatisch in eine visuelle Suchkette ein. Zusätzlich können Suchbegriffe als physische Bausteine in die Filterkette aufgenommen werden. Damit werden die vom Benutzer definierten Suchfilter durch physische Bausteine auf dem Tabletop und deren Zusammenhang in Form einer visuellen Filterkette repräsentiert. Jeder Filterbaustein gibt Aufschluss darüber, wie viele Medien sich nach dem jeweiligen Filter noch in der Filterkette befinden. Diese Information kann genutzt werden, um zu entscheiden, ob ein Filter enger oder weiter definiert werden 
muss bzw. ganz aus der Filterkette entfernt werden sollte. Die Kombination aus physischen Bausteinen zur Eingabe und der Visualisierung der Suchkette führt zu einer neuen Qualität hinsichtlich der Transparenz des Rechercheprozesses. Die jederzeitige Nachvollziehbarkeit der Auswahlkriterien, sowie die Informationen darüber, welchen Beitrag sie zum Ergebnis leisten, sind besonders hilfreich, wenn die Suchanfrage zu einer sehr großen bzw. kleinen Treffermenge führt. Der Einsatz dieser Bausteine sowie das Interaktionsdesign ermöglichen zusätzlich ein gleichberechtigtes Eingreifen mehrerer Benutzer in den Suchprozess (z. B. Filter in ihrer Ausprägung verändern oder entfernen). Auch die Ergebnisauswertung kann gemeinsam erfolgen. Das System erzeugt ein hohes Situationsbewusstsein (Social Awareness) über die jeweiligen Aktivitäten aller beteiligten Personen und unterstützt so die Zusammenarbeit. Auch können ungeübte Nutzer dem Vorgehen geübterer Nutzer oder Bibliothekare folgen und davon lernen.

Im Rahmen des Forschungsprojektes „Blended Library“ wurde auch eine Variante namens „Facet Search“ für akademische Bibliotheken entwickelt. Die Bibliothek der Universität Konstanz erprobt diesen Forschungsprototyp am Infopoint im Bibliotheksalltag. „Facet Search“ ermöglicht sehr komplexe Suchanfragen und wird daher vorwiegend für die Schulung und Beratung von Bibliotheksbenutzern eingesetzt. Frau Isabell Leibing, Sachgebietsleiterin Elektronische Medien/Mediothek der Bibliothek der Universität Konstanz, fasst die bisherigen Erfahrungen wie folgt zusammen: „Die Anwendung ,Facet Search' läuft auf einem großen Multitouch-Tisch in der Bibliothek und hat von Anfang an viel Neugier und Interesse bei Studierenden und Wissenschaftlern hervorgerufen. Der spielerische Aspekt kommt vor allem bei den Studierenden gut an, obwohl es ja ,nur' um Recherche geht. Dadurch, dass es sich um einen Prototyp und kein fertiges Produkt handelt, hat ,Facet Search“ verschiedene Einschränkungen in der Benutzung. Die intuitive Bedienung könnte insgesamt noch weiter verbessert werden, damit die Studierenden sie ohne Unterstützung durch Bibliothekspersonal selbständig und umfassend nutzen können. Die Datenlage ist noch nicht ausgereift genug, was bedingt, dass die Möglichkeiten in der Anzeige und der Weiternutzung der Ergebnisse im Wesentlichen für gedruckte und weniger für elektronische Medien möglich sind. So können zum Beispiel E-Books nicht direkt auf dem Display angezeigt und gelesen werden, was sich viele wünschen. ,Facet Search` ist daher insgesamt weniger für eine umfassende, fundierte Recherche als vielmehr zur Visualisierung von Booleschen Operatoren und Erläuterung von Recherchestrategien geeignet.“

\section{Kontextualisierung von Bibliotheksdaten mit digitalen Daten des Alltags als neuartiger Einstieg in den Rechercheprozess}

Unsere Erhebungen (siehe Kapitel 2.4) haben gezeigt, dass der Zugriff auf Medien in öffentlichen Bibliotheken häufig über aktuelle Ereignisse, Empfehlungen durch Dritte (z. B. in Form von Rezensionen) oder Diskussionen in sozialen Medien inspiriert wird. Das Forschungssystem „Expedition" stellt eine direkte Verknüpfung zwischen diesen Inspirationsquellen und dem Bestand der Bibliothek bereit und bietet den Bibliothekskunden somit einen alternativen $\mathrm{Zu}$ gang zum Medienbestand und ein neuartiges Stöbererlebnis. Das System „Expedition“ wurde für den Einsatz in öffentlichen Bibliotheken konzipiert und im Bibliotheksalltag der Stadtbibliothek Köln getestet.

Auf einem großen Touchscreen (siehe Abb.13, rechts) werden aktuelle Ereignisse in Form von Nachrichten einer lokalen Zeitung ${ }^{12}$ als interaktive Kacheln angezeigt, durch die der Benutzer frei stöbern kann. Durch Berühren einer Kachel zeigt der Anwender sein Interesse für ein bestimmtes Thema. Die Inhalte der so zentral gewählten Kachel werden durch weiterführende digitale Inhalte in Form von sich darum herum gruppierenden Kacheln angereichert (z.B. aus Quellen wie Twitter Nachrichten, ${ }^{13}$ Kartendienst, ${ }^{14}$ Wikipedia, ${ }^{15}$ Wikimedia Commons, ${ }^{16}$ Archiv für publizistische Arbeit, ${ }^{17}$ digitales Archiv des analogen Alltags ${ }^{18}$ ). Parallel dazu werden in einem digitalen Bücherregal (siehe Abb. 13, links) die zur jeweiligen Nachrichtenmeldung passenden Inhalte des lokalen Datenbestandes der Bibliothek ${ }^{19}$ in Form eines digitalen 3D-Bücherregals angezeigt. Damit wird eine Brücke zwischen dem Alltagskontext und dem Medienbestand der Bibliothek geschlagen. Das digitale Bücherregal kann nun frei durchstöbert werden und ausgewählte Bücher „fallen“ aus dem Regal auf ein kleines Lesepult unterhalb des Regals. Das ausgewählte Medium (Buch, E-Book, CD oder DVD) wird auf dem Lesepult mit zusätzlichen Informationen angereichert. Hierzu gehören Informationen zum Werk selbst und

12 Kölner Stadtanzeiger http://www.ksta.de.

13 https://twitter.com.

14 Bing Karten: http://www.bing.com/maps/.

15 http://de.wikipedia.org/.

$16 \mathrm{https}$ ///commons.wikimedia.org/wiki/Main_Page.

17 Munzinger: http://www.munzinger.de.

18 Digit: http://digit.wdr.de.

19 Stadtbibliothek Köln. 

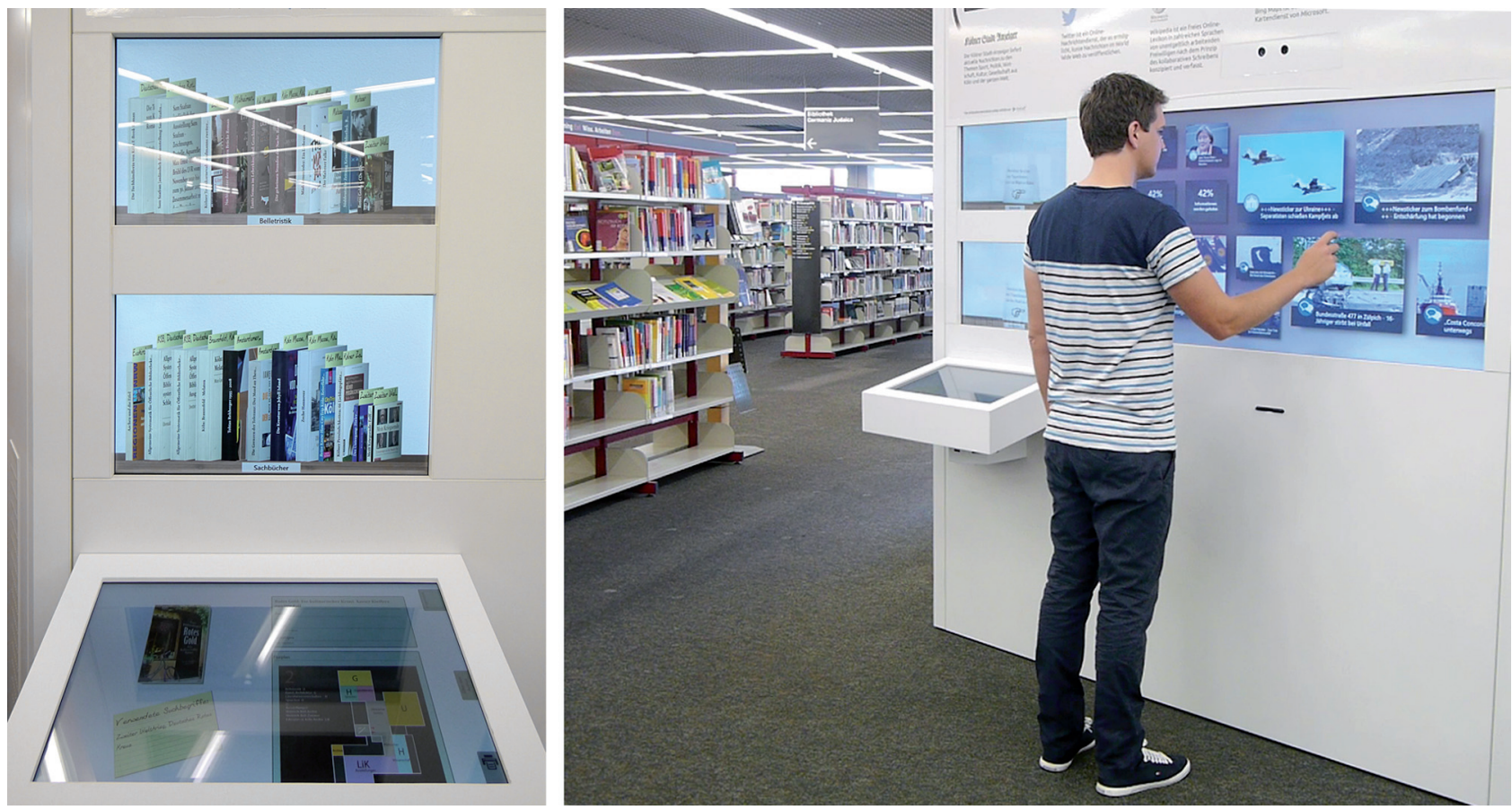

Abb. 13: Kontextualisierung des lokalen Bibliotheksbestands in der Stadtbibliothek Köln

zum Autor sowie Leserrezensionen. ${ }^{20}$ Diese Zusatzinformationen helfen dem Benutzer bei seiner Auswahlentscheidung. Somit wird auch auf der Buchebene ein Brückenschlag zu digitalen Inhalten des Alltags in Form von Leserrezensionen hergestellt und das einzelne Buch in einen erweiterten Alltagskontext gestellt. Dieses Wechselspiel zwischen dem digitalen Inhalt unserer Archive und Bibliotheken und dem größeren digitalen Alltagskontext repräsentiert durch über das Web zugängliche Inhalte eröffnet neue Zugänge zu unseren Bibliotheken und stellt sie gleichzeitig in den aktuellen Kontext.

\section{Resümee}

Im Rahmen der zwei Forschungsinitiativen „Blended Library“ (Baden-Württemberg) ${ }^{21}$ sowie „Lernort Bibliothek“ (Nordrhein-Westfalen) ${ }^{22}$ haben wir eine Reihe von Interaktionskonzepten entwickelt, welche der Zugänglichkeit zu den Inhalten von Bibliotheken eine neue Qualität verleihen. Wir haben uns dabei auf die vier Qualitäten intuitiv, kollaborativ, transparent und kontextuell fokussiert. Unserer bisherigen Erfahrungen bei der Entwicklung visueller

20 LibraryThing: https://www.librarything.com. 21 http://hci.uni-konstanz.de/blendedlibrary. 22 http://hci.uni-konstanz.de/libros.
Recherchesysteme sowie die Erkenntnisse aus umfassenden Erhebungen in wissenschaftlichen und öffentlichen Bibliotheken haben gezeigt, dass diese vier Qualitäten das Potenzial besitzen, bestehende Defizite zu überwinden und interessante neue Möglichkeiten zu bieten.

Durch die Steigerung der Transparenz des Rechercheprozesses versetzen wir die Benutzer in die Lage, die Ergebnisse der Recherche besser zu kontrollieren und zu verstehen. Hier konnten wir vor allem mit der Installation „Tiefenrausch“ in der öffentlichen Bibliothek der Stadt Köln hervorragende Einsichten gewinnen. Die visuelle Verkettung von Facetten und Stichworten - repräsentiert durch physische Suchsteine - macht den Prozess im doppelten Wortsinn „be-greifbar“. Boolesche Operatoren werden durch diese Darstellung implizit möglich, ohne sie explizit formulieren zu müssen. Unsere bisherigen Erfahrungen sind vielversprechend und basieren auf ersten teilnehmenden Beobachtungen. Aufgrund dieser Erfahrungen sehen wir auch für die Beratung von Bibliotheksnutzern durch das Bibliothekspersonal ein potentielles Einsatzfeld. Im Idealfall führt diese Beratung zur eigenständigen Nutzung der Online-Recherchemöglichkeiten und entlastet in weiterer Folge das Bibliothekspersonal. Derzeit laufen umfassende Benutzerstudien vor Ort, um die Eignung systematisch zu untersuchen. Ersten Erkenntnissen zufolge sehen die meisten Benutzer beim Erstellen von Suchanfragen einen klaren Vorteil gegenüber dem OnlineKatalog. Als Gründe hierfür wurden die physischen Suchsteine sowie deren visuelle Verknüpfung genannt. Laut 
Teilnehmer führe dies zu einem dynamischen und nachvollziehbaren Suchprozess.

Bei allen entwickelten Interaktionskonzepten ging es uns darum, die Vorzüge der Recherche in analogen Medien zu erhalten und gleichzeitig die Vorteile der Online-Recherche zu erweitern. Durch diese Vermischung von physischer und digitaler Bibliothek - deswegen sprechen wir auch von Blended Library - konnten wir die Zugänglichkeit intuitiver gestalten. Herr Oliver Kohl-Frey, Abteilungsleiter, Benutzung der Bibliothek, an der Universität Konstanz fasst die Erfahrungen bezüglich der Steigerung der Qualität der Benutzung aus Sicht der Bibliothek zusammen: „Das gemeinsame Projekt ,Blended Library“ hat eine ganze Reihe positiver Effekte mit sich gebracht. Wir haben uns als Bibliothek intensiv mit den Ideen und Anregungen der Wissenschaft auseinandergesetzt und viel über Usability gelernt. Für die beteiligten Bibliothekarinnen und Bibliothekare sind Touch-Systeme ein Stück weit selbstverständlich geworden, vorhandene Hemmschwellen wurden abgebaut. Die Erfahrungen mit einzelnen Prototypen waren so gewinnbringend, das wir in der Folge mit dem ,Hybrid Bookshelf‘ ein Produkt haben entwickeln lassen, das wir nun mit großem Erfolg einsetzen können und das auch für interessierte andere Institutionen kostenfrei zur Verfügung steht.“

Unsere Erfahrungen im Zusammenhang mit der technologischen Unterstützung von kooperativen Rechercheformen sind erfolgversprechend. Sie zeigen, dass hier großes Potenzial für wissenschaftliche Bibliotheken besteht, da Teamarbeit und die gemeinsame Generierung von Wissen besser unterstützen werden kann. Insbesondere unsere Erfahrungen bei der Nutzung des Forschungsprototyps „TwisterSearch“ haben gezeigt, dass die Möglichkeiten zur gemeinsamen Erstellung und Strukturierung der Suchbegriffe zu einer besseren Abdeckung des Suchraums führen. Gleichzeitig lernen die Benutzer durch Beobachtung anderer Teammitglieder verschiedene Recherchestrategien kennen und können sich bei Problemen besser gegenseitig unterstützen. Die unmittelbare Präsentation und Diskussion der gemeinsam gefundenen Rechercheergebnisse vertieft das gemeinsame Verständnis für den Suchraum. Dies ermöglicht in der Folge den Benutzern, präzisere bzw. weitergehende Suchbegriffe $z u$ formulieren, und so den Rechercheprozess unter Nutzung des Wissens aller Teilnehmer zielgerichteter fortzusetzen. Als wichtiger Nebeneffekt entsteht bei allen Teammitgliedern ein besseres, gemeinsames Verständnis des zu bearbeitenden Sachverhalts (z. B. Projektfragestellung, Referat, Übung).

Die Kontextualisierung von Inhalten unserer Bibliothek mittels digitaler Daten des Alltags erscheint uns gerade für öffentliche Bibliotheken ein vielversprechender An- satz, um neue Zugangswege zu den Bibliotheksinhalten zu ermöglichen. Hier konnten wir mit der Installation „Expedition“ in der öffentlichen Zentralbibliothek der Stadt Köln vielversprechende Einsichten durch erste teilnehmende Beobachtungen gewinnen. Durch die explizite digitale Präsentation von tagesaktuellen Inhalten wird eine Alternative zu heute üblichen „Aufstellern“ mit aktuellen Büchern geboten. Einerseits werden die Benutzer zum Stöbern in diesen aktuellen Inhalten animiert und durch die Integration verschiedener und interessanter Datenquellen (neben aktuellen Nachrichten eben auch Inhalte aus sozialen Medien, Nachschlagewerken, digitalen Archiven oder Kartendiensten) bietet dieser Zugang schon einen eigenen Mehrwert und Reiz für den Bibliotheksbesucher. Die parallel erfolgende Kontextualisierung der Bibliotheksinhalte durch ein anschauliches digitales 3D-Bücherregal bietet naturgemäß mehr Flexibilität als ein herkömmlicher physischer Aufsteller. Die technologische Herausforderung besteht allerdings darin, bei den vorgeschlagenen Medien die passende Auswahl zu treffen. Dies ist in hohem Maße von der verfügbaren Datenqualität abhängig. Eine gewisse „Unschärfe“ wird von Benutzern (im Gegensatz zu Bibliothekaren) toleriert. Allerdings darf nicht der Eindruck der Beliebigkeit entstehen, da dann das Vertrauen in die präsentierten Inhalte verlorengeht. Hier die richtige Balance zu finden, gehört zu den technologischen Herausforderungen. Derzeit werden umfassende Benutzerstudien vor Ort durchgeführt, um die Akzeptanz dieser neuen Zugangsform systematisch zu testen. Erste Erkenntnisse ergaben ein interessantes Bild bezüglich der Wahrnehmung der vorgeschlagenen Medien: Während der eine Teil der Benutzer sich eine präzisere Auswahl der Medien gewünscht hätte, wurde eben diese Unschärfe vom anderen Teil durchaus geschätzt.

Wir verbinden mit den hier beschriebenen Forschungsergebnissen die Hoffnung, dass sie Bibliotheken und Forscher zur Gestaltung neuer Formen der Zugänglichkeit inspirieren. Unsere Ideen mögen dazu erste Denkanstöße liefern. Abschließen möchten wir mit den Erfahrungen der Direktorin der Bibliothek der Universität Konstanz Frau Petra Hätscher: „Die Resonanz aus der bibliothekarischen Öffentlichkeit ist sehr positiv. Die Themen in den Projekten rund um Blended Library greifen einen Bedarf der Praxis auf, der aus der Praxis heraus mangels Mitteln - nicht bedient werden kann. Insofern sind die Kooperationen in den „Blended Library“-Projekten zwischen Forschung und Informationsinfrastruktureinrichtung ein Erfolgsmodell. Die Weiterentwicklung des Projektes „Blended Shelf“ zur Anwendung „Hybrid Bookshelf“ ist ein Beispiel von gelungenem Transfer von der Forschung in die Wirtschaft unter Berücksichtigung der 
Auflagen der öffentlichen Hand (Stichwort: Open-SourceProdukte und freie Nachnutzbarkeit).“

\section{Literatur}

Björneborn, L.: Serendipity Dimensions and Users' Information Behaviour in the Physical Library Interface. Information Research 13 (1) (2008) paper 370. http://InformationR.net/ir/13-4/pa per370.html.

Blended Library (2015): http://hci.uni-konstanz.de/blendedlibrary. Eibl, M.; Löbel J.-M.; Reiterer, H.: Grand Challenge „Erhalt des digitalen Kulturerbes“. In: Informatik Spektrum 2015. DOI: 10.1007/ s00287-015-0898-5. Print ISSN: 0170-6012, Online ISSN: 1432-122X, Heidelberg: Springer Verlag.

Evans, B.; Chi E.: Towards a Model of Understanding Social Search. In: Proceedings of the 2008 ACM Conference on Computer Supported Cooperative Work (CSCW ‘08). New York 2008, S. 485-494.

Hartson, R.; Pyla, P.: The UX Book. Process and Guidelines for Ensuring a Quality User Experience. Burlington, Mass. 2012.

Hearst, M.: Search User Interfaces. Cambridge 2009.

Heilig, M.; Rädle, R.; Reiterer, H.: Die Blended Library: Benutzerorientierte Verschmelzung von virtuellen und realen Bibliotheksdiensten. In: Bekavac, Bernard; Schneider, René; Schweibenz, Werner (Hrsg.). Benutzerorientierte Bibliotheken im Web. Berlin 2011.

Gerken, Jens; Heilig, Mathias; Jetter, Hans-Christian; Rexhausen, Sebastian; Demarmels, Mischa; König, Werner A.; Reiterer, Harald: Lessons Learned from the Design and Evaluation of Visual Information Seeking Systems. In: International Journal on Digital Libraries (2009) S. 49-66.

Jetter, H.-C.; Gerken, J.; Zöllner, M.; Reiterer, H.; Milic-Frayling, N.: Materializing the Query with Facet-streams: A Hybrid Surface for Collaborative Search on Tabletops. In: Proceedings of the SIGCHI Conference on Human Factors in Computing Systems (CHI '11). ACM, New York 2011, S. 3013-3022.

Kleiner, E. (2013). Ein realitätsbasierter Ansatz zur Präsentation und Exploration von Bibliotheksbeständen. Master Thesis, University of Konstanz. http://eprints.rclis.org/22434/

Kleiner, E.; Rädle, R.; Reiterer, H.: Blended Shelf: Reality-based Presentation and Exploration of Library Collections. In: Proceedings of CHI EA '13 (2013) S. 577-582. doi:10.1145/2468356.2468458.

Kohl-Frey, O.: Die Öffnung der digitalen Magazinbibliothek: Entwicklungen an der Universität Konstanz zwischen Summon und Hybrid Bookshelf. In: Bibliotheksdienst 48 (2014) doi:10.1515/bd2014-0098.

Libros (2015): http://hci.uni-konstanz.de/libros.

Marchionini, G.: Toward Human-Computer Information Retrieval Bulletin. In: Bulletin of the American Society for Information Science. June/July (2006). http://www.asis.org/Bulletin/Jun-06/m archionini.html.

Morris, M.: Collaborative Search Revisited. In: Proceedings of the 2013 Conference on Computer Supported Cooperative Work (CSCW ‘13). New York 2013, S. 1181-1192. doi:10.1145/ 2441776.2441910

Rädle, R.; Weiler, A.; Huber, S.; Jetter, H.-C.; Mansmann, S.; Reiterer, H.; Scholl, M. H.: E-Book Meets Tabletop: Using Collaborative Visualization for Search and Serendipity in Online Book Repositories. In: Proc. of BooksOnline '12. New York 2012, S. 3. doi:10.1145/2390116.2390120.
Reiterer, H.: Blended Interaction - Ein neues Interaktionsparadigma. In: Informatik Spektrum (37) (2014) S. 459-463, doi:10.1007/ s00287-014-0821.

Reiterer, H.: Blended Interaction - Konzepte für die Bibliothek der Zukunft. Vortrag vom 6.3.2015, Tagung „Die Zukunft der Wissensspeicher“. Gerda von Henkel Stiftung. Zugriff zum Vortrag unter: http://www.lisa.gerda-henkel-stiftung.de/blended_inter action_konzepte_fuer_die_bibliothek_der_zukunft?na $v_{-} i d=5604$.

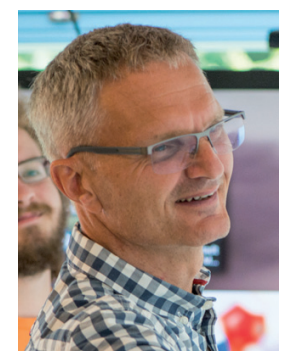

\section{Harald Reiterer}

Universität Konstanz

Fachbereich Informatik und Informationswissenschaft

AG Mensch-Computer Interaktion

Universitätsstr. 10

D-78457 Konstanz

harald.reiterer@uni-konstanz.de

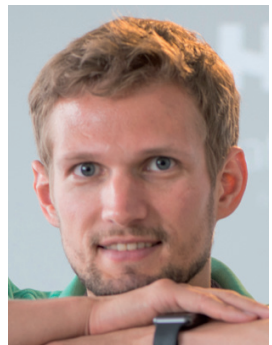

\section{Roman Rädle}

Universität Konstanz

Fachbereich Informatik und Informationswissenschaft

AG Mensch-Computer Interaktion

Universitätsstr. 10

D-78457 Konstanz

roman.raedle@uni-konstanz.de

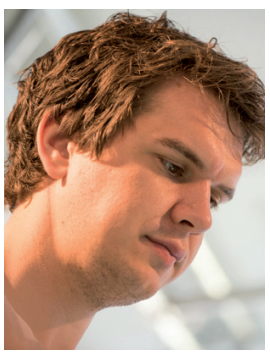

\section{Simon Butscher}

Universität Konstanz

Fachbereich Informatik und Informationswissenschaft

AG Mensch-Computer Interaktion

Universitätsstr. 10

D-78457 Konstanz

simon.butscher@uni-konstanz.de

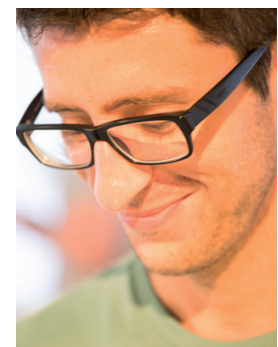

Jens Müller

Universität Konstanz

Fachbereich Informatik und Informationswissenschaft

AG Mensch-Computer Interaktion

Universitätsstr. 10

D-78457 Konstanz

jens.mueller@uni-konstanz.de 